\title{
REDEFINING THE ROLE OF THE NURSE ACADEMIC IN PRACTICE: A PILOT STUDY
}

\section{Research Paper}

Worldwide, health services are interested in supporting the speedy adoption of research findings into practice. To promote the translation of research into practice, a university in the South of England along with a partner NHS Trust piloted a new role - Translation Fellow (TF). This article describes and analyses the experience of piloting this role. This paper outlines the successes achieved as a result of this partnership between a university and a healthcare organisation as well as describing the challenges involved in establishing such a role. The successes included submitting a joint abstract to a conference; collaboratively developing articles for publication; organizing a visit overseas to compare similar services; co-designing a database to assist in collecting data for service planning and research, and setting up a 'one click access' web space populated with evidence informed material to support the work of the clinical staff. The pilot acted as a proof of concept in which the TF role demonstrated its potential. Additional roles are already being established nationally.

\section{INTRODUCTION}

The need to integrate research, education and service delivery is emphasised frequently in public policy (Walshe and Davies, 2013). Interventions to speed up translation of new scientific knowledge from 'bench to bedside' are required (Department of Health, 2011) and are of international interest (Committee on Quality of Healthcare in America IOM 2001). In nursing, this interest in research translation is also influenced by a long-standing concern about the theory/practice gap. Redefining the nurse lecturer role is frequently proposed as a way of closing this gap (Williamson, 2004) and debates about the clinical role of nurse lecturers have intensified since the transfer of nursing education into Higher Education (Barrett, 2007). Interest has also been growing as to how best to enable and support clinical staff to engage with and use research in their practice (Squires et al, 2011). Consequently, the development of new ways to address the challenges involved in research translation is of importance to nurse education nationally and internationally.

To promote the translation of research into practice, a university in the South of England along with a partner healthcare organisation piloted a new role - Translation Fellow (TF). The TF role was conceived of as a vehicle for enabling research translation by being a visible and useful academic presence to help address clinical concerns. This article describes and analyses the experience of implementing the TF role. Piloting the TF role as a participatory action research process (Reason and Bradbury, 2008). It presents the successes achieved as a result of this partnership as well as the challenges involved in establishing such a role.

\section{BACKGROUND}

How best to speed up the implementation of research findings into practice in healthcare ? This has long been recognised as an important question globally (Committee on Quality of Healthcare in America IOM 2001; Walshe and Davies, 2013). In nursing, a further and related concern is the difference between what student nurses are taught in university and what they experience happening in clinical areas. This concern is often referred to as the theory/practice 
gap to which refocusing the nurse lecturer role is frequently proposed as a solution (Williamson, 2004). Consequently, the development of new ways to address the challenges involved in research translation is of importance to nurse education nationally and internationally (Squires et al, 2011).

A university in the South of England along with a partner NHS Trust developed and piloted a new role -Translation Fellow (TF) - to support the translation of research into practice for the benefit of student learning. At conception, it was intended that the TFs should have a dual function supporting the implementation of research into practice and acting as conduits to research teams by identifying questions for further research. To assist them in enacting this dual function the original intention was for the TFs to engage together as a Community of Practice and for them in turn to establish interdisciplinary Communities of Practice in the practice area.

Two TFs were recruited from existing academic staff. Both had PhDs and had strong links to research groups. GL had worked for several years in a research institute where the "bench to bedside' ethos was evident in the strong relationship between laboratory/scientific researchers and clinicians. This environment allowed for meaningful translational research to be undertaken and applied. She had also worked in a clinical school of nursing where collaborative research as highly valued and routine (Lee \& Metcalf, 2009). EK's expertise lies in translation of bench science to clinical practice, an activity that is publication as well as classroom led. He has experience in running clinically-based research utilisation programmes which aim to enable senior clinical staff to understand research and instigate research activity of their own. EK also has expertise in the development of the practice and theory of online technologies in pedagogical and clinical practice.

The @Home service was designed to offer an alternative model of care to hospital based treatment. It provides integrated care for people in their own homes with a wide range of conditions. It supports early discharge from hospital as well as preventing unnecessary admissions. The @Home team provide daily visits up to four times a day for 3-7 days and offers intensive nursing, physiotherapy and occupational therapy supported by domiciliary visits by a consultant or @Home GP. The team meets daily to review the patient's progress. A rapid response 'out of hours' urgent/crisis nursing care service is also available and provides prompt clinical support and nursing care at short notice, through proactive visits, or in response to an unscheduled request.

At conception, it was intended that the TFs should have a dual function supporting the implementation of research into practice and acting as conduits to research teams by identifying questions for further research. To assist them in enacting this dual function the original intention was for the TFs to engage together as a Community of Practice and for them in turn to establish interdisciplinary Communities of Practice in the practice area.

The @Home service was designed to provide a minimum of 80-100 'beds' for patients in a home-ward service in an inner-city setting. It included admission avoidance, early discharge 
and case management. In effect this was a new department within Community Services, with four Home Wards which included nursing, social work, GP, therapy and pharmacy. The supporting infrastructure included a dedicated service management and clinical leadership role, clinical practice development, quality assurance, evaluation and research a clinical nurse practitioner liaising with each hospital. The initial implementation plan had identified a wide range of conditions which would be suitable for the service to pick up and therefore divert from hospital admissions, ranging from post-operative support for cancer patients, to serious asthma referrals from GPs, for example. Referrals were made to the service to a hospital- based team member and a strict inclusion criteria was applied to patients (need for acute home-based care for no more than 5 days, over 18 years, living locally and agreeing to be seen by the team in their home).

The university commissioned the lead author (AM) in December 2013 to prepare the selected academic staff for the Translation Fellow role, support them in enacting it and articulate the resulting 'know how' in a way that enables it to be shared with others.

This paper critically explores the experience of implementing the TF role to support the translation of research into practice, and captures the learning about what worked well and what was problematic in order that others might learn from it (Berwick, 2008; Stevens, 2012). Ethics The purpose of this inquiry was to develop, test and refine the design of the TF role (i.e its purpose was 'service improvement' rather than research and consequently it did not require ethical approval) (NHS Health Research Authority, 2015). In an action inquiry of this type, those involved; the stakeholders and authors of this paper, were exploring situations they are part of, and to which they 'belong', and so already have ethical responsibilities towards the others involved. The inquiry therefore is an extension and an intensification of those same ethical relations (Munn-Giddings and Winter, 2013). Care was taken throughout to ensure that the rights and well-being of all those involved were kept central. All the co-authors have been closely involved in the project and in the preparation of this article.

\section{METHODS}

The project took the form of an action inquiry in that it was concerned with studying a social situation, in this case the implementation of a new role, with a view to improving the quality of action within it (Reason and Bradbury, 2008). The primary purpose of the inquiry was to support this local project but it has produced some practical. The pilot study was constructed as a small participatory action research study. Participatory Action Research as defined by Reason and Bradbury (2008) is research that seeks to understand a social situation as well as to change or transform that situation. It involves a collective approach to knowledge generation using iterative cycles of action and reflection. All stakeholders were facilitated to reflect on their experience at various stages in the pilot and to use the insights and learning this generated to inform the next steps. In this pilot the aim was to understand: how to successfully implement a TF role in a clinical area; and by the action of introducing the role, to increase the implementation of research findings in that clinical area and generate questions for further clinical research. The primary purpose of the pilot study was to support local implementation of the TF role. The participatory action research process has also produced some useful 
knowledge about the design and implementation of a new role for nurse academics that is likely to be of interest to others. TheConsequently, this article is concerned withexplores the research process of inquiry as well as its findings. It benefits from the authors' practical involvement and offers a careful and close yet critical examination of the experience of implementation and its impact (Munn-Giddings and Winter, 2013).

Data collection

The stakeholders in this initiative were senior managers and staff and students of the university and the clinical area where the TFs were working and the TFs. The data collected included conversations (chance and purposeful) with stakeholders, observations of and reflections on meetings with the TFs and other key stakeholders, and documents such as PowerPoint presentations about the initiative created by the TF, emails, Steering Group and smaller task group meeting notes. From January 2014 until July 2015 there were eight steering group meetings, eight sessions with xxAM and the TFs, one session facilitated by xxAM with the clinical staff and one of the TFs, three meetings with internal stakeholders and numerous conversations and emails.

Analysis Emerging themes and interpretations were tested out with stakeholders at Steering Group meetings. Further testing out and refinement occurred in the process of co-constructing this article.

\section{FINDINGS}

Terminology The original intention was to establish a Community of Practice (Wenger \& Rayner, 2015) of TFs and the TFs in turn to establish Communities of Practice in the service they were allocated to. In this pilot both terms, 'Translation Fellows' and 'Communities of practice' proved problematic. Neither term secured immediate understanding with all stakeholders. Translational Research is a term used in the research and policy literature to describe implementing research findings into practice, however it is not a term that is necessarily familiar to clinical staff. For example, several of them suggested that the term 'practice development' might be more appropriate. The following explanatory paragraph was developed in April 2014: "Research Translation Fellows have a passion for practice and tackling the theory practice divide. Research Translation Fellows help bring research from 'bench to bedside'. Research Translation Fellows appraise the evidence base for practice, demystify research for clinicians and support them to consider how and what research might inform their practice. Research Translation Fellows legitimise clinician engagement in research, role model how to use evidence in practice and support student learning. Research Translation Fellows work towards making research part of clinicians' day to day practice and mindsets."

Communities of practice as defined by Wenger and Rayner (2015) are groups of people who share a concern or a passion for something they do; , in this case research translation, and who interact regularly to learn how to do it better. The aim of the project was to use a community of practice approach, as advocated by Rowley et al (2012), to actively support implementation of the new role, encourage knowledge sharing about the TF implementation process and build and sustain the TF role identity. 
TFs saw the term 'community of practice' as unhelpful and confusing jargon, in part perhaps because the nursing in question was 'community nursing' and community nursing is the term used to describe nurses who work in people's homes and 'community' seen as the place where non-hospital care is delivered. The Community of practice terminology therefore was potentially off-putting and acting as a barrier to understanding and thus engaging with the wider audience of clinicians and academics. Nevertheless, the underpinning principles of translational research and communities of practice were realised as demonstrated in the collaborative process the TFs engaged in with their clinical colleagues and the outcomes that were achieved.

The role The role of the TF was to act as a link to bring about changes to the curriculum and work with research teams to identify questions for further research; to provide a vehicle for mutual learning and develop educational materials and to support students undertaking their placements. The key objectives in the role description were making a significant contribution to the translation of research findings into clinical practice and generating enthusiasm for research in clinical staff and supporting them in translation activities. The outputs expected included writing academic papers, presenting at conferences and leading reviews to facilitate improvement. To achieve this TFs were expected be a "visible and influential academic presence in the relevant clinical area" and within their workload, 40 days allocated annually for the role.

Clinical staff suggested that it was important that the TFs had: "A certain type of character, have to be pushy, self-confident and able to engage with people". The TFs emphasized the importance of perseverance:

"From my part I think perseverance rather than being pushy is probably a better descriptor of how access was gained, relationship development then was aided by finding areas of immediate clinical need that would help facilitate the relationship and understanding rather than the actual translational activity itself. Using these "soft touch" introductory methods access was gained and dialogue was initiated, now with maturation of the relationship more "translational" interactions can and have been identified. Culture change in clinical practice to help staff utilise research has to be identified by staff as a necessity rather than being imposed upon them."

The TFs and the clinical staff also saw personal credibility as vital to success and considered that this credibility is derived from the individual's personal clinical expertise.

Working to different timetables and priorities The TFs and xxAM were appointed in December 2013. In the first 6 months, the clinical staff were understandably focused on the highly complex task of developing and delivering a new service to meet ever-changing local demands. During 2014 (and since) the service continued to innovate, develop and grow, for example taking on a 'first response' role with the London Ambulance Service and developing a hyperemesis treatment protocol for pregnant women. The service is a high-profile initiative, with the potential to transform the culture and practice of community services more generally, and so was constantly under scrutiny from a variety of stakeholders. Unlike traditional community based nursing, the service runs 7 days a week with staff covering withereither a $8 \mathrm{am}$ to $8 \mathrm{pm}$ shift to $11 \mathrm{am}$ to $11 \mathrm{pm}$ shift. 
Recruitment and induction of front-line@Home staff with therequire a wide range of competencies and advanced assessment skills required to meet the complexity of service user need and. Recruiting such staff in thesufficient numbers to sustainsupport the growth of the service was a constant necessity and therefore a pre-occupation for the senior managers and the clinical team. The workload pressures on the TFs were significant too. In tandem with their TF role, they were expected to secure research grants and publish in journals with high impact factors as well as to teach and assess student work at under-graduate and post-graduate levels.

As the clinical service was evolving, organisational structures were changing, as were the roles and responsibilities of the clinical staff whom TFs were trying to engage with. Individual team members changed position or were seconded onto other projects within the Trust. The constant operational pressures on the clinical staff meant that meetings between the TFs and clinicians frequently had to be rearranged, and only the most senior of the NHS staff could attend Steering Group meetings. All of which made developing relationships more complex. Developing the TF role at the same time as establishing a new service meant that service development was inevitably the first priority for the clinical staff. The newness of the service provided opportunities too, for example one of the key challenges for the clinical staff was ensuring that the new community based services were supported by evidence and development of appropriate guidelines (for examplesuch as use of intravenous diuretics in heart failure patients), something the TFs could assist with. As one of the senior clinical staff said "It's a privilege working with academics" and emphasized the value of having access to a resource such as a TF who could look at the evidence, knew what evidence was available and could help establish useful national and international links and relationships, and assist the clinicians publish their work.

Building relationships There was high level commitment to the TF roles from the university and the NHS Trust but establishing relationships at the frontline between the TFs and the clinicians in the NHS

Trust took a long time: this. This was partly because the service ran formfrom two locations and staff were often out of the office. In the beginning, the only opportunities for face-to- face discussions between the university and clinical staff were at the Steering Group meetings. All stakeholders were represented at these meetings, which were large (the membership was 15 with 8 to 12 people attending the meetings) and had a formal agenda. This format was not ideal for building the types of working relationships that an innovation of this sort requires. Early discussions in the Steering Group tended to have an almost competitive edge with each side keen to show the value of what they were already doing and the many things they could offer the partnership. As the trust between people began to build the tone became more conversational and one year in, two members of the group, each from a different organisation, presented some joint work they had been engaged in and referred to the collective as "we". The conversation flowed around and across the table with each member regardless of the organisation they are employed by, freely voicing their own opinions and perspectives rather than defending an organisational view. This was also facilitated by one of TFs visiting the @Home site and engaging with staff and assisting the set-up of a more user-friendly database. Gaining access and making connections New services and new roles are often subject to significant scrutiny by stakeholders. The clinical service was under tremendous pressure to 
demonstrate its impact on reducing admissions to the acute hospitals from the locality and ensuring high levels of patient satisfaction. This occurred at the time when there were access issues across the UK with

heavy pressures on acute services and emergency departments. This contributed to the time taken to gain access to the clinical teams and to enable meetings in small groups between the TFs and the clinical staff. The NHS senior team were very protective of their clinical staff and acted as gatekeepers to the service:

"It's been such a frenetic few months, building relationships, feeling under scrutiny, imbalance of staff - we've got bands 5 and 3 but no band 7s, it's the timing ...".

For most of the first year, the service was underrunning below capacity in terms of staff and senior staff were directly engaged in delivering clinical care which made organizing meetings difficult.

An invite to a Clinical Implementation Group for the TFs to talk about their role was secured five months in. However, the invitation was confined to only part of the meeting rather than to the whole meeting and there was no invite to engage directly with the front-line clinical staff, which made the TFs feel disheartened:

"I've tried to be interactive and accommodating but I just feel I'm being told - you can't join our group - it should be a partnership".

An important breakthrough in establishing this sense of partnership and connection was the wish for the new service to be available to students for placement - a mutually beneficial common purpose. This enabled direct meetings with the senior clinical staff in the service andwhich provided the opportunity to explore potential areas of synergy and mutual benefit such as. This included supporting senior clinical staff in accessing master's degree studies and introducing them to the resources available internationally such as protocols developed for similar service in Australia. The TFs completed the required practice placement audit and student nurses commenced four week clinical placements in November. These placements were evaluated very positively by the students (Lee et al., 2015) and the staff were very proud of the feedback.

Areas of synergy and mutual benefit Much of the discussion in the TF meetings, and the Steering Group meetings was about what the TFs could offer and what the clinical team wanted or might find useful. Early ideas suggested by the TFs included pharmacology and medication management including evaluating quality of life and measuring cognition. However, the major concerns expressed by the clinical team were demonstrating the impact of the service and staffing, how to recruit appropriately skilled individuals and develop an appropriate organizational culture. Further ideas were offered by the academics on the wider Steering Group which tended to reflect their research expertise as well as focusing on student experience and learning. Some concern about role was expressed by the clinical team, one for example saying "We are not there to be your research subjects". There was additional complexity in that another academic institution had been commissioned to undertake an evaluation of the NHS service. However, by explicitly revisiting what the TFs could offer and what the clinical team would find useful in the TF meetings and at the Steering Group, gradually over the months, several themes began recurring as potential areas of synergy and mutual benefit. These were: 
- Managing (re)Hydration in patients own homes and nursing homes - including administration of subcutaneous and IV fluids.

- Managing Heart Failure in patients own homes - including administration of furosemide

- Understanding the development staff need and supporting the induction and education of the new recruits to the service.

- Research based policies and protocols to be made available as smartphone/tablet Apps to support practice

- Developing and embedding a new service requires a significant culture change amongst the practitioners involved and stakeholders in the system such as patients and referring agencies. Understanding the nature of this culture change and how to enable/encourage it.

- Providing literature on other similar service models, contact details of such services and access to protocols.

As one of the TFs said:

"I think we are there now in terms of trust and understanding, so that we can help clinical staff of all bands own small data projects that need to be done anyway in terms of audit. This activity will help them understand research. Together with the journal club and online materials recently identified for development these will give them the tools they will need to understand and facilitate translation of research into practice."

Successes

Collaboratively developing success criteria is a core element of community of practice work (Wenger \& Rayner, 2015). Following discussions with the TFs and the Steering Group members the potential success criteria suggested in March 2014 included the TFs engaging in a shared endeavour with clinicians; establishing systems for collecting and analysing data on the service and who is using it for service and research purposes; developing a shared publicationpublications (Lee and Titchener, 2016; Lee et al, 2017) and several conference presentations. By July 2015, these had all been achieved and more as listed below:

$\square$ Successful submission of a joint abstract to a conference $\square$ Collaborative development of two articles for a professional journal one describing the service and the other the student placement experience.

$\square$ Developing a detailed database for data entry allowing real time data to be collated with multiple variables.

$\square$ Collaborative work on refining the demographic data stored on the 'home service clients using to maximise its potential for service planning and its utility for research purposes.

$\square$ Setting up 'one click access' web space populated with evidence informed material to support the work of the clinical staff such as ECG e-learning materials and a literature review on rehydration therapy in older patients.

$\square$ Commencement of a Churchill Fellowship application to explore similar services in Australia and New Zealand.

Supporting the TF development The original intention was to establish a community of practice as a safe and supportive space for the TFs to assist them in implementing their 
new role. Regular meetings were scheduled between the TFs and XxAM for the first 6 months of the project for this purpose. In the early meetings, time was spent considering the terminology and expectations of the TF role. The TFs evident enthusiasm for the role and its potential provoked wide ranging and ambitious discussions about what had been done that could be translated into practice. Then, given the delays the TFs were experiencing in negotiating access, discussions moved on to how to refine and improve the 'offer' the TFs could make to their clinical colleagues and identifying individuals the TFs could make contact with in the NHS team to begin to build relationships at the front line.

On reflection, although there was some value in the external facilitation offered by xxAM in drawing key points together and confirming action points at the end of each session, using this resource differently to support relationship building across the two organisations, rather than focusing it on the TFs might well have been more fruitful. Offering some workshops for clinical staff and TFs outside of the formal Steering Groups early on may have assisted in the establishment of a common purpose and identification of areas of synergy and mutual benefit. DISCUSSION There is a long history of developing new nursing roles in the UK and internationally (Read et al, 2001; Bridges et al, 2007). Roles designed particularly to bridge the theory practice gap also have a long history. (Williamson, 2004). Embedding new roles successfully, particularly those crossing organisational boundaries, is recognised to be difficult (Drennan and Goodman, 2011). Much of the literature on nurse lecturers' engagement with clinical areas is written from the perspective of supporting nurse lecturers to maintain their skills and credibility and provide a link with clinical practice (Barratt, 2007). The TF role had a very different purpose - promoting research translation, yet many of the challenges experienced in establishing the role such as gaining access, managing expectations regarding the competing demands on the TFs and their time, were similar to and reflect the findings of the broader literature on new role development.

Even though the two organisations involved are strategic partners in the local health economy and as partners collaborate on many initiatives and developments, the individual services and departments involved in this development had not collaborated previously. Group development takes time (Tuckman, 1965) and this needs to be acknowledged and planned for. The clinical service with which the TFs were working was a new service and was constantly evolving throughout this period as well as recruiting new staff for the multidisciplinary team. Consequently, the time it took for the TFs to gain access to clinical colleagues may be linked to the stage of development the service was in when the new TF role was introduced. As one of the TFs said:

"I think the time it took for us to gain meaningful access was possibly more to do with the stage we were introduced. This was a newly evolving service and clinical staff did not have time for themselves, let alone with a person with a relatively poorly defined role of TF.... If there was anything I would have done differently, I would have not initiated our role at the stage we did. We should have been brought in either from the initial stages of team development so that we were an integral part of the team from the beginning or been introduced to the team probably in 6 months' time from now as they would have had time to consolidate their initial practices and work force." 
Understanding the demand on the clinical staff is important as is their capacity to engage with research. The need for the clinical staff in this pilot to focus on embedding a new service meant that time was a major hurdle in translating research into practice. It also meant the TFs had to be flexible when planning meetings:

"It is important that the TFs visit the service so that the dynamics of the workplace can be seen and appreciated. The recipe for success is patience and a common goal and once this has been established, the journey is more satisfying."

As was hoped there has been much learning from the process. Time is a critical factor in developing and embedding a new role and such an intervention needs a minimum of two years to begin to reap significant rewards. Previous literature on integrating clinical academics has highlighted the importance of just being around and being useful as a way of becoming accepted and learning about the realities of practice. In an increasingly virtual world, many of the interactions take place by email. This raises questions about when and how best to establish a presence.

The TFs also saw personal credibility is vital to success and considered that this is derived from the individual's personal clinical expertise, from the careful selection of post-holders and matching with sites, and from talking and meeting together in smaller, more informal groups to build trust and keep the momentum going. Once again, the time needed for this should not be underestimated, as it is through this that they and the teams found a common purpose in fostering the two-way translation between research and practice.

The approach clearly had its limitations as a pilot involving two TFs from the same university in one clinical service. The issues may therefore be different in other organisations and working with services which are at a different stage in their development. Furthermore, by their nature such interventions are not going to be on a scale which would enable quantitative analysis. However, many of the experiences echoed existing work on new role development cross organisational boundaries.

CONCLUSIONS As questions are currently being asked about the role of universities in developing nurse education, and suggestions are made that nurse academics should be judged on whether and how their findings are translated into clinical care (Melnyk, 2014), TFs provide a useful reminder of the skills nurse academics can bring to clinical practice and vice versa. The collaboration between the clinical team and the TFs, although it took time to develop, became a highly productive partnership. It is extremely unlikely that the outputs achieved would have occurred without the TF role, and more TFs are planned for other clinical areas. Although to date the role has only been piloted in one university and healthcare organisation, itsgiven that nurse academics across the globe struggle with getting research findings implemented in practice, the impact to dateof this pilot suggests that itthe TF role merits wider discussion and further testing in other settings and services.

\section{REFERENCES}

Barrett, D (2007) The clinical role of nurse lecturers: Past, present, and future. Nurse Education Today, Volume 27, Issue 5, $367-374$ 
Berwick, D.M. (2008). The science of improvement. Journal of the American Medical Association, 299(10), 1182-1184.

Bridges, J., Fitzgerald, L., and Meyer, J. (2007) New workforce roles in healthcare: exploring the longer term journey of organisational innovations. Journal of Health Organisation and

Management 21, (4/5), 381-392

Committee on Quality of Healthcare in America IOM (2001) Crossing the quality chasm: a new health system for the 21st century. Washington, DC, National Academies press

Cooksey, D. (2006) A review of UK health research funding. London, HMSO

Department of Health (2011) Innovation, health and wealth. London: Department of Health

Drennan, V., and Goodman, C. (2011) Sustaining innovation in the healthcare workforce: a case study of community nurse consultant posts in England. [Online] BMC Health Services Research 11, 200. < http://www.biomedicalcentral.com/1472-6963/11/200> [8/9/12]

Lee, G., Metcalf, S. (2009) Building research capacity through a hospital-based clinical school of nursing. Nurse Education Today, 29 (3), 350-356

Lee, G., Sakone, P., Mulhall, H., Kelleher, K., Burnett, K. (2015) Using hospital-at-home to reduce admissions. Nursing Times, 111 (36/37), 12-15.

Lee GA, Titchener, K (2016). The Guy's and St Thomas's NHS Foundation Trust @home service: an overview of a new service. London Journal of Primary Care. DOI:

10.1080/17571472.2016.1211592

Lee, G, Pickstone, N, Jose Facultad, Titchener, K (2017) The future of community nursing: Hospital in the Home. British Journal of Community Nursing 22(4) 650-654

Melnyk, B.M. (2014) Speeding the translation of research into evidence-based practice and conducting projects that impact healthcare quality, patient outcomes and costs: The "so what" outcome factors. Worldviews on Evidence-Based Nursing, 11(1),pp.1-4.

Munn-Giddings, and C. Winter, R. (2013) A handbook for action research in health and social care. London, Routledge

NHS Health Research Authority (2015) Determine whether your study is research http://www.hra.nhs.uk/research-community/before-you-apply/determine-whether-yourstudy-is-research/

Read, S., Lloyd-Jones, M., Collins, K., McDonnell, A., Jones, R. Doyal, L, Cameron, A., Masterson, A., Dowling, S., Vaughan, B., Furlong, S., and Scholes, J. (2001) et al. Exploring new roles in practice: implications of developments within the clinical team (ENRiP). University of Sheffield: Sheffield. https://www.academia.edu/3270447/Exploring_New_Roles_in_Practice_ENRiP_ Reason, P., \& Bradbury, H. (Eds.). (2008). Sage Handbook of Action Research: Participative inquiry and practice (2nd ed.). London : Sage Publications.

Squires, J.E., Estabrooks, C.A., Gustavsson, P., Wallin, L. (2011) Individual determinants of research utilization by nurses: a systematic review update. Implementation Science. 6:1 doi:10.1186/1748-5908-6-1

Stevens, K.R. (2012). Delivering on the promise of EBP. Nursing Management, 3(3).

Philadelphia: Lippincott, Williams \& Wilkins, Inc.

Thomson, L., J. Schneider and N. Wright (2013). 'Developing communities of practice to support the implementation of research into clinical practice', Leadership in Health Services, 26(1): 2033. 
Tuckman, Bruce (1965). "Developmental sequence in small groups". Psychological Bulletin 63 (6): 384-99. doi:10.1037/h0022100. PMID 14314073.

Walshe, K., \& Davies, H. T. (2013). Health research, development and innovation in Wenger-Trayner, E. (2015) ConmunitiesCommunities of Practice a brief introduction http://wenger-trayner.com/theory/

Williamson, G. (2004) Lecturer practitioners in UK nursing and midwifery: what is the evidence? A systematic review of the research literature. Journal of Clinical Nursing 13, 787- 795 